\section{Nocardiosis in \\ Immunocompromised Patients on Alternative Pneumocystis Prophylaxis}

\author{
Alfredo G. Puing, David J. Epstein, Niaz Banaei, \\ Aruna K. Subramanian, Anne Y. Liu
}

Author affiliations: City of Hope National Medical Center, Duarte, California, USA (A.G. Puing); Stanford University School of Medicine, Stanford, California, USA (A.G. Puing, D.J. Epstein, N. Banaei, A.K. Subramanian, A.Y. Liu)

DOI: https://doi.org/10.3201/eid2710.210620

Prophylactic trimethoprim/sulfamethoxazole (TMP/SMX) prevents Pneumocystis jirovecii pneumonia and nocardiosis in immunocompromised patients but sometimes is avoided because of purported allergies or side effects. Of 25 immunocompromised patients receiving alternative prophylaxis in whom nocardiosis developed, 16 subsequently tolerated TMP/SMX treatment. Clinicians should consider TMP/SMX allergy evaluation and rechallenging to assess patient tolerance.

$\mathrm{T}_{\mathrm{i}}^{\mathrm{r}}$ rimethoprim/sulfamethoxazole (TMP/SMX) is the drug of choice for Pneumocystis jirovecii pneumonia (PJP) prophylaxis in immunocompromised patients (1). Second-line prophylactic agents include atovaquone, dapsone, pentamidine, and clindamycin with pyrimethamine. Alternative agents can be less effective than TMP/SMX at preventing PJP and opportunistic infections caused by Listeria monocytogenes, Toxoplasma gondii, and Nocardia spp. Prophylactic TMP/SMX is sometimes avoided because of a prior adverse drug reaction or when patients are receiving drugs that have potentially overlapping toxicities. Nonetheless, secondline PJP prophylaxis regimens can increase the risk for opportunistic infections, such as nocardiosis (2). Most nocardiosis occurs in patients with impaired cell-mediated immunity; TMP/SMX is the cornerstone of standard therapy (3). We describe a series of nocardiosis cases in immunocompromised patients who were receiving alternative or no PJP prophylaxis because of TMP/SMX avoidance. We provide the reasons for TMP/SMX avoidance and proportion of patients who subsequently tolerated TMP/SMX.

We conducted a retrospective chart review at Stanford Hospital (Stanford, CA, USA) for patients with nocardiosis diagnosed during January 1, 1998-January 28, 2020. We included patients avoiding TMP/SMX for PJP prophylaxis in whom nocardiosis was identified on culture or by molecular techniques, such as $16 \mathrm{~S}$ rRNA PCR-based assay. We used Stanford Hospital's protocols for defining immunocompromised status requiring PJP prophylaxis. We collected baseline demographic, clinical, microbiological, and outcome information, including immunocompromising condition, PJP prophylaxis indication and agent, reason for TMP/SMX avoidance, and TMP/SMX rechallenge outcome, if performed. This study was approved by Stanford University's Institutional Review Board (approval no. 54959).

During the study period, nocardiosis developed among 25 immunocompromised patients deliberately avoiding TMP/SMX. Most (68\%) patients were female; median age of patients was 55 years. Among the 25 patients, 7 (28\%) were lung transplant recipients, 6 (24\%) had undergone allogeneic hematopoietic cell transplantation (HCT), 5 (20\%) were heart transplant recipients, and 7 (28\%) had other immunocompromising conditions (Appendix Table, https://wwwnc.cdc.gov/EID/article/27/10/21-0620.App1.pdf). At diagnosis, 15 $(60 \%)$ patients were taking atovaquone, $4(16 \%)$ inhaled pentamidine, $3(12 \%)$ dapsone, and $3(12 \%)$ no antimicrobial drug prophylaxis.

Thirteen (52\%) patients were not taking TMP/ SMX because of a reported history of allergy, 6 because of concern for cytopenia (24\%), and 3 because of elevated creatinine (12\%). TMP/SMX was avoided in 1 patient for elevated transaminases, 1 for gastrointestinal intolerance, and 1 for unstated reasons. Among 10 patients with a TMP/SMX allergy label who attempted challenge or desensitization, 7 (70\%) tolerated the drug; nonsevere rash developed in the other 3 patients. Among 10 patients avoiding TMP/SMX prophylaxis for nonallergy reasons, $9(90 \%)$ tolerated TMP/SMX when rechallenged. Overall, TMP/SMX introduction was attempted in $20 / 25$ patients; $80 \%$ successfully tolerated the drug, and $20 \%$ had mild, reversible adverse effects (Figure).

In this retrospective case series, 16/25 (64\%) patients who had nocardiosis while deliberately avoiding TMP/SMX prophylaxis ultimately were treated with TMP/SMX. Immunocompromised patients often are prescribed alternative drugs to TMP/SMX prophylaxis because of concerns over side effects or allergic reactions (4). However, desensitization or rechallenge could enable a substantial proportion of patients to safely take TMP/SMX for prophylaxis. In our study, $70 \%$ of patients with a history of TMP/ 


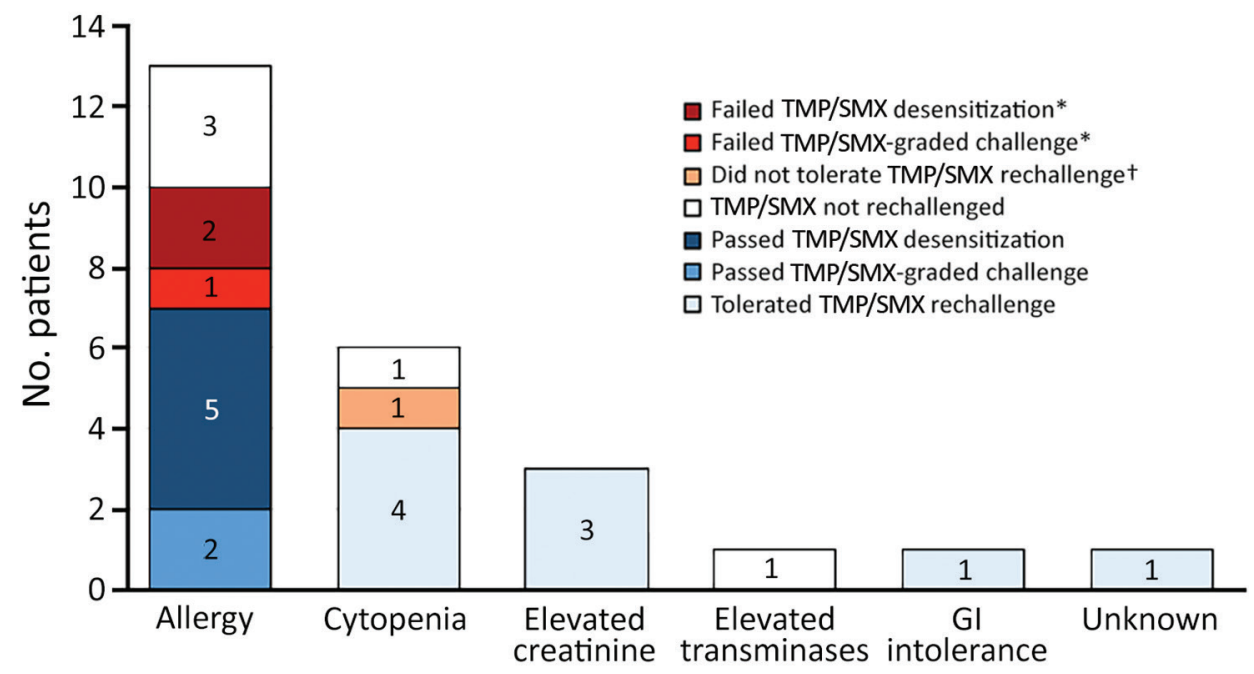

Figure. Reasons for TMP/ SMX avoidance and TMP/SMX rechallenge outcomes among immunocompromised patients in whom TMP/SMX prophylaxis for Pneumocystis jirovecii pneumonia prophylaxis was avoided, Stanford, California, USA. *Failed TMP/SMX introduction because of rash or Gl symptoms that were not severe. †Developed intractable nausea and vomiting after TMP/ SMX was introduced and did not tolerate rechallenge. GI, gastrointestinal; TMP/SMX, trimethoprim/sulfamethoxazole
SMX allergy tolerated a TMP/SMX graded challenge or desensitization when attempted, and $90 \%$ of patients avoiding TMP/SMX prophylaxis for nonallergy reasons tolerated TMP/SMX when rechallenged. Our results concur with findings from a study that showed $74 \%$ of kidney transplant recipients who underwent TMP/SMX rechallenge had no recurrence of adverse drug reactions (5).

TMP/SMX prophylaxis might decrease the incidence of nocardiosis in immunocompromised patients. In a retrospective review of HCT recipients with nocardiosis, most $(12 / 15)$ cases occurred in patients receiving alternate PJP prophylaxis (2). Other studies have questioned the efficacy of TMP/SMX prophylaxis in preventing nocardiosis in HCT or solid organ transplant recipients $(6-8)$.

Taken together, these findings suggest that rates of this highly pathological infection might be reduced by systematically reevaluating TMP/SMX avoidance and reconsidering prophylactic TMP/SMX. Consulting with an allergist can detect contraindications, such as severe cutaneous adverse reactions, and opportunities for challenge or desensitization. Patients with a history of maculopapular rash, cytopenia, or increased creatinine with TMP/SMX might tolerate reintroduction. Electronic medical records can be designed to prompt revisiting whether TMP/SMX avoidance is appropriate (9).

The first limitation of our study is that we only included immunocompromised patients from a single healthcare system; our findings might not be generalizable to other settings. Second, some immunocompromised patients with nocardiosis possibly were not included in our cohort; although defining the incidence of nocardiosis would be informative, the intent of our study was to describe consequences of unnecessary TMP/SMX avoidance. Third, specifics of desensitization or graded challenge protocols were not consistently documented and thus might not be uniform.

Despite these limitations, our study shows that most patients in whom nocardiosis developed while avoiding TMP/SMX prophylaxis later tolerated TMP/SMX treatment. Future research should prospectively evaluate the risks and benefits of TMP/ SMX reintroduction in immunocompromised patients who have had a prior adverse reaction. In conclusion, our findings suggest that revisiting TMP/ SMX avoidance could prevent nocardiosis cases.

A.G.P. developed the research question, designed the data collection, compiled and analyzed the data, contributed to writing the manuscript, developed the figure, revised the manuscript; A.Y.L. developed the research question, designed the study, supervised data collection and cleaning, contributed to data analysis and manuscript preparation; D.J.E., N.B., and A.K.S reviewed the manuscript and provided expert input; all authors approved the final version of the manuscript to be published.

\section{About the Author}

Dr. Puing is an infectious diseases specialist at City of Hope National Medical Center. His main research interests center on evaluating the impact of infections following solid organ and hematopoietic stem cell transplantation, and the efficacy of antiinfective prophylaxis protocols.

\section{References}

1. Stern A, Green H, Paul M, Vidal L, Leibovici L. Prophylaxis for Pneumocystis pneumonia (PCP) in non-HIV 
immunocompromised patients. Cochrane Database Syst Rev. 2014;2014:CD005590. https://doi.org/10.1002/

14651858.CD005590.pub3

2. Shannon K, Pasikhova Y, Ibekweh Q, Ludlow S, Baluch A. Nocardiosis following hematopoietic stem cell transplantation. Transpl Infect Dis. 2016;18:169-75. https://doi.org/10.1111/tid.12499

3. Wallace RJ, Septimus EJ, Williams TW, Conklin RH, Satterwhite TK, Bushby MB, et al. Use of trimethoprimsulfamethoxazole for treatment of infections due to Nocardia. Rev Infect Dis. 1982;4:315-25. https:/ / doi.org/ 10.1093/clinids/4.2.315

4. Epstein DJ, Benamu E, Subramanian AK. Use of alternative agents for prevention of opportunistic infections in heart and lung transplant recipients. Clin Infect Dis. 2018;67:1637-9. PubMed https://doi.org/10.1093/cid/ciy397

5. Urbancic KF, Ierino F, Phillips E, Mount PF, Mahony A, Trubiano JA. Taking the challenge: A protocolized approach to optimize Pneumocystis pneumonia prophylaxis in renal transplant recipients. Am J Transplant. 2018;18:4626. PubMed https://doi.org/10.1111/ajt.14498

6. Coussement J, Lebeaux D, van Delden C, Guillot H, Freund R, Marbus S, et al. Nocardia infection in solid organ transplant recipients: a multicenter European case-control study. Clin Infect Dis. 2016;63:338-45. https://doi.org/10.1093/cid/ciw241

7. Hemmersbach-Miller M, Stout JE, Woodworth MH, Cox GM, Saullo JL. Nocardia infections in the transplanted host. Transpl Infect Dis. 2018;20:e12902. https:/ / doi.org/ 10.1111/tid.12902

8. Filice GA. Nocardiosis in persons with human immunodeficiency virus infection, transplant recipients, and large, geographically defined populations. J Lab Clin Med. 2005;145:156-62. https://doi.org/10.1016/j.lab.2005.01.002

9. Baneman E, Kim N, Rana M, Renteria AS, Steinberg AS, Jakubowski RM, et al. Optimizing the use of trimethoprimsulfamethoxazole for prevention of PCP and opportunistic infections in allogeneic hematopoietic cell transplant recipients. J Clin Oncol. 2018;36:138. https:/ / doi.org/ 10.1200/JCO.2018.36.30_suppl.138

Address for correspondence: Alfredo G. Puing, Division of Infectious Diseases, Department of Medicine, City of Hope National Medical Center, 1500 E Duarte Rd, Modular 1 West, Duarte, CA 91010, USA; email: apuing@coh.org

\section{Autochthonous Case of Rickettsia slovaca Infection in Russia}

\author{
Ruslan F. Sayfullin, Nadezhda E. Perekopskaya, \\ Ludmila S. Karan, Nadezhda N. Zvereva, \\ Muhammad A. Sayfullin \\ Author affiliations: Municipal Clinical Hospital No 52, Moscow, \\ Russia (R.F. Sayfullin); Pirogov Russian National Research \\ Medical University, Moscow (R.F. Sayfullin, N.N. Zvereva, \\ M.A. Sayfullin); Infectious Clinical Hospital No. 1, Moscow \\ (N.E. Perekopskaya); Central Scientific Research Institute of \\ Epidemiology, Moscow (L.S. Karan); Gamaleya Institute of \\ Epidemiology and Microbiology, Moscow (M.A. Sayfullin).
}

DOI: https://doi.org/10.3201/eid2710.204621

We describe an autochthonous case of Rickettsia slovaca infection in a man 35 years of age from Russia who had tickborne lymphadenopathy. We used ELISA and quantitative PCR testing to further identify DNA and confirm diagnosis. Physicians in Russia should consider similar diseases in differential diagnoses after tick bites.

$R$ ickettsia slovaca was isolated in Dermacentor marginatus ticks in 1968 in Slovakia and recognized as a Rickettsia species with unknown pathogenicity. In 1997, a study described the first laboratory-confirmed case of Rickettsia slovaca infection in a human (1). R. slovaca has been detected in ticks in many countries in Europe, including the Mediterranean region. Human cases of syndromes that can be caused by $R$. slovaca, including tickborne lymphadenopathy (TIBOLA), Dermacentorborne necrosis-erythema-lymphadenopathy (DEBONEL), and scalp eschar and neck lymphadenopathy after tick bite (SENLAT) have been reported $(2,3)$. $R$. slovaca has been detected in ticks in 4 of 85 regions of Russia (Figure), and 1 imported case of $R$. slovaca infection was reported (4-7). The aim of our study was to describe an autochthonous case of $R$. slovaca infection in a man in Russia.

In May 2019, a 35-year-old male resident of Russia with an unremarkable medical history sought treatment for eschar on the skin of his right shin, painful and enlarged inguinal lymph nodes, rash, pain in his right knee, and severe fatigue. Before onset, he was in a rural village in the Voronezh region of Russia for 8 days, where he had contact with domestic animals and later noticed an insect bite near the location of the eschar. He reported no history of foreign travel in the previous 6 months. 